\title{
SEGURIDAD E HIGIENE EN EL SECTOR DE LA CONSTRUCCIÓN: EL COMIENZO DEL FIN DE UNA INDIGNIDAD
}

\author{
(THE BEGINNING OF THE END OF AN INDIGNITY)
}

Manuel Garnacho Villarrubia, Secretario General

FEMCA-Unión General de Trabajadores

Fecha de recepción: 14-I-93

$750-10$

\section{RESUMEN}

Es una presentación de la realidad en aspectos de prevención, seguridad y salud laboral en el sector de la construcción y en el proceso de ejecución de obra planteándose la intervención en el "riesgo evitable y excesivo" de "las Administraciones y los Promotores", de "los Técnicos Diseñadores del Proyecto", de las "Empresas Constructoras" y, por último, de "los Trabajadores".
SUMMARY

In a presentation of the reality in terms of prevention, safety and labour health in the construction sector and in the process of works execution considering the intervention in event of the "inevitable and excessive risk" of "the Administrations and Promotors", of "the Technical Designers of the Project", of the "Construction Companies" and lastly of "the workers".
Por una parte, es preciso destacar la importancia del sector de la construcción en nuestro país, y por otra poner de manifiesto las características que definen esta actividad como peculiar.

En primer lugar el sector de la construcción representa entre el 9 y 10 por ciento del PIB español y el $10 \%$ de la población activa de nuestro país.

Por otro lado se trata de una actividad móvil, que cambia con el tiempo y el espacio mientras se realiza la obra de construcción, a diferencia de otras actividades que son fijas y estáticas.

El trabajo se suele realizar al aire libre, en las vías públicas y en la mayoría de las ocasiones interfiriendo con otros servicios y a merced de las inclemencias del tiempo.

Los materiales empleados como matería prima son muy variados, desde muy ligeros a muy pesados, manipulándose a nivel del suelo y a grandes alturas.
A estas circunstancias hay que añadir el gran número de personas que intervienen en el proceso, y la diversidad de oficios y empresas que concurren a un mismo tiempo, en el mismo lugar.

Todas estas características hacen de la construcción un sector sometido a situaciones de riesgo, que originan que

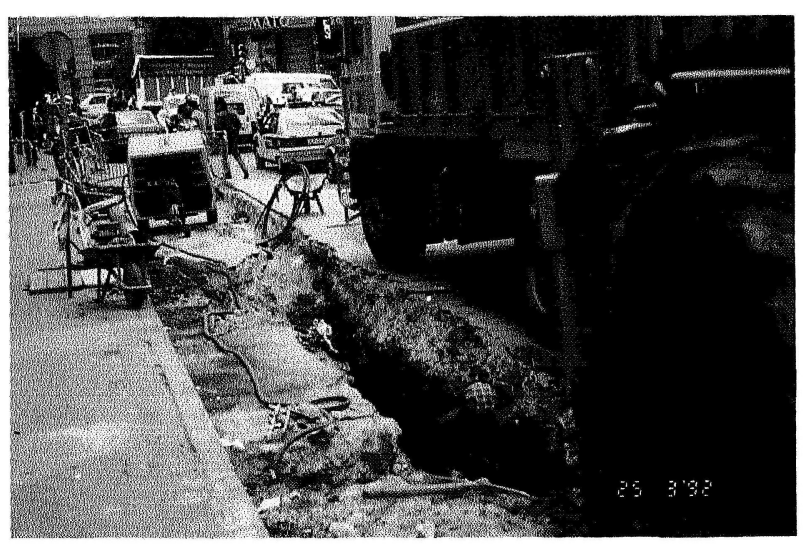

Sin vallas, sin casco, sin entibación y bajo el peso de las máquinas. 


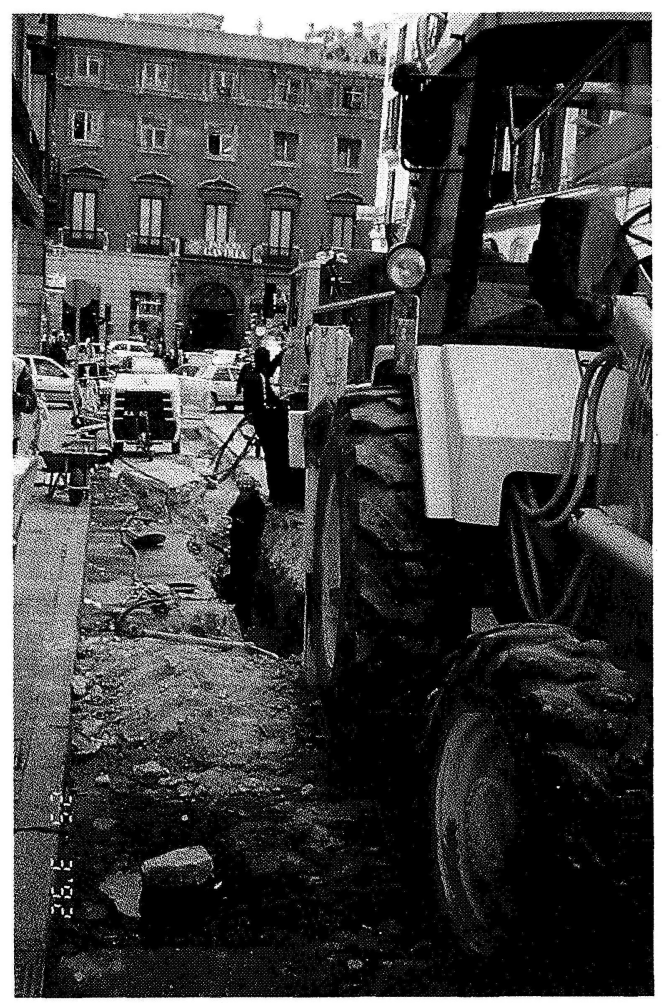

Sin vallas, sin casco, sin entibación y la máquina se sube encima de la zanja.

en esta actividad se produzca el mayor número de accidentes. La mayoría de los riesgos pueden preverse, a priori, durante la realización del proyecto y durante el estudio económico que realiza la empresa constructora antes de iniciar la obra.

Nos encontramos ante un sector en el que se lucha por la dignidad. Cuando en países vecinos, muchos jóvenes escogen por vocación el integrarse profesionalmente en el sector de la construcción, en el nuestro se producen llegadas transitorias.

Para dignificar el sector, habrá que suprimir los puntos negros que hoy lo hacen indigno y, no cabe duda, que la siniestrabilidad es uno de ellos.

Estadísticamente año tras año, por cada mil trabajadores asalariados en el sector de la construcción, la tasa de siniestrabilidad es el doble que en el resto de los sectores productivos del país. Esto, por tanto, demuestra la indignidad a la que nos referimos.

Hemos clasificado en cuatro grandes grupos las causas y los causantes de esas altas cifras de siniestrabilidad.

\section{A. En primer lugar las administraciones y promotoras.}

En cuanto a la responsabilidad que tienen, conociendo la legislación en materia de salud laboral a nivel internacional, nos atrevemos a decir que nuestros textos legislativos se situarían a la cabeza.

El problema surge por la escasa puesta en práctica o el escaso cumplimiento de esta legislación. Por ejemplo, en 1986 después de la lucha emprendida desde nuestra Federación, se obtuvo la promulgación de un nuevo texto, el Real Decreto 555/1986. Al año siguiente, en 1987, en una Comisión Técnica de la OIT, Estados como Alemania o Dinamarca nos pidieron que les facilitáramos la totalidad del Decreto para intentar hacer una legislación parecida.

Este Real Decreto 555/86 se incumplió sistemáticamente, incluso hubo campañas de desobediencia civil de colectivos corporativos. Nos referimos a Colegios de Arquitectos, a los que el Real Decreto otorgaba la responsabilidad de establecer los proyectos de seguridad que se tenían que aplicar en las obras. También el Colegio de Ingenieros de Caminos se negaba a cumplir las atribuciones que les otorgaba el mencionado Real Decreto.

Ante estas presiones, en 1990 se modificó el Real Decreto ofreciéndose los arquitectos técnicos para que el Decreto pudiera salir adelante, asumiendo la autoría de los estudios de seguridad. Sin embargo, se rompió algo que el Decreto establecía: que el autor del proyecto de la obra tuviera que ser el responsable del proyecto de seguridad.

En cuanto a la Administración, como promotora, hemos denunciado sistemáticamente que existiendo el Real Decreto 555/86 demasiados proyectos, por no decir todos, salían de los ministerios (fundamentalmente del MOPU hoy MOPT) sin llevar el proyecto de seguridad. Por tanto, difícilmente la Administración podría hacer cumplir su legislación por promotoras privadas, si ella misma, en su calidad de promotor, no la cumplía. Si bien la situación era ésta, a partir del 20 de noviembre de 1991, se firmó un protocolo entre el MOPT y el MTSS paliándose esa falta de cumplimiento de los proyectos de Seguridad. Hoy los proyectos que surgen desde la Administración (MOPT) llevan en su mayoría el proyecto de Seguridad. 
Otro incumplimiento por parte de la Administración se refiere a las Corporaciones Locales, a las que el Decreto $555 / 86$ obliga a conceder las licencias de obra, siempre que el proyecto vaya acompañado, a su vez, del proyecto de seguridad. Cuando lo cumplen lo hacen únicamente como registro; así es muy corriente que se fotocopie el proyecto de seguridad de otra obra y se adjunte con el proyecto a realizar, por lo cual creemos que es importante controlar que el proyecto de seguridad, que se presenta, corresponda auténticamente a la obra que se va a realizar.

También existen incumplimientos por parte de la Administración en su papel de controlador y sancionador. La Inspección de Trabajo ha mejorado bastante en sus actuaciones, si bien carece de medios humanos suficientes, debido al elevado grado de incumplimiento de las leyes sobre seguridad en nuestro país.

Es muy importante el papel de colaboración que estamos desarrollando desde los Sindicatos con la Inspección de Trabajo.

Siendo la Inspección de Trabajo el órgano sancionador del Estado en esta materia, no es el recaudador y en muchos casos Hacienda no recauda las sanciones que impone el Ministerio de Trabajo, por tanto muchas empresas prefieren correr el "riesgo" de incumplir la legalidad vigente.

\section{B. En segundo lugar encuadramos a los Técnicos Diseñadores del proyecto.}

Otro problema es la planificación, que hace que tengamos que construir con plazos demasiado cortos que imponen altos ritmos de trabajo.

Estamos acostumbrados a trabajar sobre proyectos que en cualquier país de nuestro entorno no pasarían de ser un anteproyecto, pero en ningún caso un proyecto de ejecución. Sobre esto, también nuestra legislación dice que para obtener las licencias y permisos de obra, basta con el proyecto básico. Este proyecto, en muchos casos, poco tiene que ver con el proyecto de realización.

Según un estudio realizado por la Fundación Europea para la mejora de las condiciones de vida y de trabajo, con sede en Dublín, se demuestra que solamente un ter- cio de los accidentes que se producen en la construcción tienen como origen las condiciones de realización de la obra. El resto de los accidentes tienen su origen en las diferencias prácticas del proyecto y en los materiales.

\section{La tercera de las causas son las Empresas Constructoras.}

En ningún país europeo es tan fácil como en el nuestro, el convertirse en empresario o constructor, no se exige ninguna profesionalidad, por lo que en el censo se encuentran unas 200.000 unidades empresariales, muchas de las cuales tienen vidas muy cortas (nacen y mueren para una obra o una promoción).

Esperamos que estas incorrecciones se puedan solventar a través de nuestra colaboración con la Dirección General de la Vivienda y de Arquitectura, en la redacción del nuevo texto de la ley de ordenación de la Edificación, que esperamos vea pronto la luz.

Las empresas además de incumplir la legislación en materia de Seguridad e Higiene, lo hacen también sobre la jornada laboral.

Existe la contratación fraudulenta en gran parte de nuestro sector y también el fenómeno de los "falsos autónomos", que supone prestamismo laboral. Esto está extendido por toda la geografía, y hoy incluso se está extendiendo por Europa.

Se trata de empresas que despiden a sus trabajadores y les indican que se den de alta en el régimen de la Seguridad Social como autónomos, asegurándoles la empresa trabajo a destajo. Más del $25 \%$ de los trabajadores en activo del sector, lo hacen bajo la figura de "falsos autónomos".

Existen maquinarias y materiales inadecuados. A través de un accidente que se produjo por la caída de la aguja de una grúa, se descubrió una red de empresas que compraba en Francia grúas achatarradas, y bajo una capa de pintura las llevaban a las obras como grúas en perfecto estado. Asimismo, se produce un incumplimiento en los controles que el Ministerio de Industria tendría que realizar sobre la maquinaria usada. 

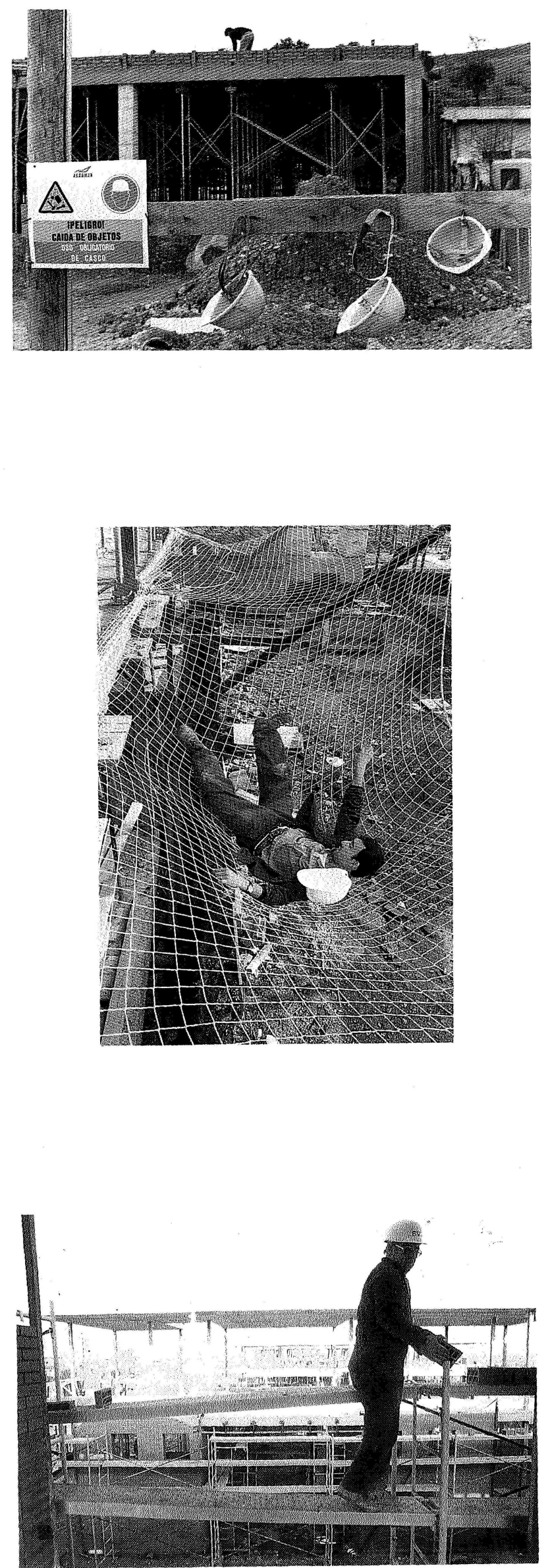

Por otra parte, la aplicación legislativa de la "Ergonomía", es muy escasa.

La mala organización de las obras es también uno de los factores influyentes en la siniestrabilidad. Los Órganos de Dirección de las empresas constructoras, responsables de las mismas, desconocen las condiciones en las que se están ejecutando sus obras, delegando en personas cuya formación y conocimiento en materia de Seguridad es nula.

La subcontratación es un fenómeno tan normal, que ha convertido a las empresas constructoras en gestoras. Éstas únicamente llevan la dirección de la obra y subcontratan la ejecución de la misma a gran cantidad de pequeñas o medianas empresas.

El $85 \%$ de los trabajadores en activo lo están con contratos precarios.

\section{Como último grupo tenemos a los Trabajadores.}

En primer lugar por la falta de formación y profesionalidad.

Como se dijo al principio estamos en un sector "recoge todo". Todo aquel que no tiene especialidad, se destina a construcción. Ejemplo, inscripción en el INEM.

$\mathrm{Al}$ ser un sector poco atractivo para los jóvenes, tenemos unas plantillas profesionales envejecidas, cuya edad media está muy por encima de la de los sectores de producción. Eso conlleva mayores riesgos, ya que los trabajadores no están en condiciones para prever los accidentes y protegerse de ellos, debido a que durante muchos años se han acostumbrado al riesgo y pasan un poco de él.

Entendemos que no se puedan hacer jornadas excesivas ni trabajos a destajo que, científicamente, está demostrada su repercusión en la salud y, por tanto, en la seguridad.

Para corregir esto, desde nuestra Federación estamos denunciando todas las irregularidades detectadas, tanto a las administraciones, como a la Inspección de Trabajo. Hacemos campañas de mentalización de nuestros propios afiliados. Hemos confeccionado juegos de diaposi- 


\section{SECTOR CONSTRUCCIÓN}

POBLACION OCUPADA - ACCIDENTES SEGÚN GRAVEDAD

\begin{tabular}{|c|c|c|c|c|c|c|}
\hline & 1986 & 1987 & 1988 & 1989 & 1990 & 1991 \\
\hline $\begin{array}{l}\text { POBLACIÓN } \\
\text { OCUPADA }\end{array}$ & 827.000 & 925.100 & 1.020 .300 & 1.133 .900 & 1.220 .400 & 1.273 .500 \\
\hline $\begin{array}{l}\text { TOTAL CON BAJA } \\
\text { Acc. Trabajador } \\
\text { - Enfer. profes. }\end{array}$ & $\begin{array}{r}71.092 \\
97\end{array}$ & $\begin{array}{r}88.013 \\
120\end{array}$ & $\begin{array}{r}104.258 \\
172\end{array}$ & $\begin{array}{r}125.951 \\
186\end{array}$ & $\begin{array}{r}145.533 \\
262\end{array}$ & $\begin{array}{r}146.395 \\
350\end{array}$ \\
\hline $\begin{array}{l}\text { Accidentes/ } \\
\text { Población ocupada }\end{array}$ & $8,6 \%$ & $9,5 \%$ & $10,2 \%$ & $11,1 \%$ & $11,9 \%$ & $11,5 \%$ \\
\hline $\begin{array}{l}\text { LEVES } \\
\text { - Acc. Trabajador } \\
\text { - Enfer. profes. }\end{array}$ & $\begin{array}{r}69.084 \\
94\end{array}$ & $\begin{array}{r}85.633 \\
115\end{array}$ & $\begin{array}{r}101.370 \\
162\end{array}$ & $\begin{array}{r}122.860 \\
182\end{array}$ & $\begin{array}{r}142.193 \\
248\end{array}$ & $\begin{array}{r}143.149 \\
345\end{array}$ \\
\hline $\begin{array}{l}\text { GRAVES } \\
\text { - Acc. Trabajador } \\
\text { - Enfer. profes. }\end{array}$ & $\begin{array}{r}1.794 \\
3\end{array}$ & $\begin{array}{r}2.156 \\
5\end{array}$ & $\begin{array}{r}2.586 \\
10\end{array}$ & $\begin{array}{r}2.765 \\
4\end{array}$ & $\begin{array}{r}3.006 \\
14\end{array}$ & $\begin{array}{r}2.923 \\
5\end{array}$ \\
\hline $\begin{array}{l}\text { MORTALES } \\
\text { - Acc. Trabajador } \\
\text { - Enfer. profes. }\end{array}$ & $\begin{array}{c}214 \\
-\end{array}$ & $\begin{array}{c}224 \\
-\end{array}$ & $\begin{array}{l}302 \\
-\end{array}$ & $\begin{array}{l}325 \\
-\end{array}$ & $\begin{array}{l}334 \\
-\end{array}$ & $\begin{array}{l}323 \\
-\end{array}$ \\
\hline
\end{tabular}

Al tiempo que el número de trabajadores aumentaba, el número de horas trabajadas también era mayor, así pues aumenta la accidentabilidad, sin embargo en 1991 el número de horas trabajadas disminuye y es por esa razón por lo que disminuyen los accidentes, ya que el tiempo de exposición al riesgo es menor.

tivas para enseñar cuáles son los riesgos de las zanjas, andamios, cuadros eléctricos, etc. Es decir, los riesgos más frecuentes, con objeto de que el trabajador lo detecte y sepa cómo protegerse. También colaboramos en la búsqueda de soluciones, no sólo a nivel nacional sino a nivel internacional, ya que está reconocido en todos los países que el Sector de la construcción conlleva un alto riesgo.

Por consiguiente nosotros estamos luchando por la dignificación del sector en:

A.- Que el joven o el trabajador que venga de otro sector reciba una formación profesional adecuada.

B.- Que se mejoren las condiciones de defensa de salud laboral.

C.- Que haya estabilidad en el empleo.

Creemos que estos son los tres fundamentos básicos para que el sector de la construcción empiece a ser digno en España.
La primera piedra en este sentido, creemos que ha sido el Convenio General de la Construcción.

Como segunda piedra, el texto que tienen que consensuar las partes firmantes del convenio en materia de seguridad, que ha de ser una norma que recoja el control de la seguridad y de la salud desde los mismos trabajadores a través del sindicato. Ha de incluir mecanismos que a la vez vigilen el cumplimiento de toda la normativa en la materia.

Como ejemplo, en el año 1992 se han finalizado las obras de las Olimpíadas y la Expo, importantes tanto por el volumen como por el número de trabajadores, así como por la colaboración entre promotores (COOB, Sociedad Expo 92), empresas y sindicatos, que introdujeron los medios oportunos para evitar el riesgo de accidentes y se consiguieron bajar las habituales tasas de siniestrabilidad a menos del $50 \%$.

También los cometidos que ha de desarrollar la "Fundación Laboral de la Construcción", recientemente crea- 
da entre la Asociación Empresarial y nuestros Sindicatos, y que son encaminados a:

- formar adecuadamente a los trabajadores (manuales y técnicos);

- estudiar y realizar programas concretos de protección de la salud y mejora de las condiciones de trabajo;

- y estabilizar el empleo mediante la homogeneización de los costes laborales sustituyendo las primas de an- tigüedad en las empresas por primas de permanencia en el sector.

Este sustancial cambio de las tradicionales -y sistemáticamente colectivas- relaciones laborales, en España en general, y en la Construcción en particular, que ha posibilitado sustituir la vieja y franquista Ordenanza Laboral por un Convenio General del sector, constituye, a nuestro juicio, el fundamento del proceso de dignificación y profesionalización de este importante sector de producción.

\section{publicación del ICCET/CSIC}

INSPECCION DE OBRAS DAÑADAS POR CORROSION DE ARMADURAS

El presente Manual va dirigido principalmente a técnicos especializados y laboratorios que tienen que intervenir en el dictamen de la situación de deterioro de estructuras de hormigón armado dañadas por corrosión de armaduras.

Comienza con un resumen recordatorio de los factores principales a los que se pueden deber los daños prematuros por corrosión de armaduras, para seguir con algunas indicaciones de cómo se deben realizar las inspecciones, y de los ensayos y la metodología que se recomienda realizar para poder dictaminar con precisión las causas de daño.

A continuación se hacen una serie de comentarios sobre la vida residual de estructuras dañadas, sobre el riesgo de corrosión futura, el seguimiento necesario de una estructura reparada y una breve enumeración de métodos de reparación y consideraciones básicas a tener en cuenta en la recomendación de un determinado método. Se aporta una breve relación bibliográfica.

Finalmente se incluyen en forma de ficha la descripción de algunos casos de corrosión de armaduras detectados en nuestro país.

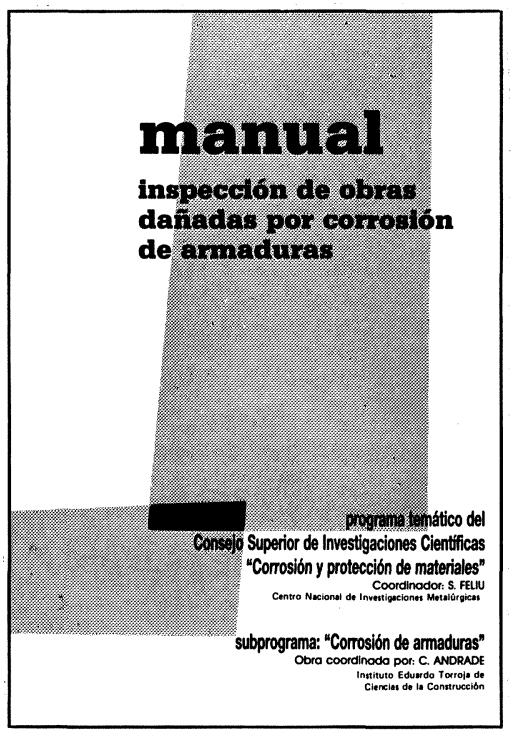

